\title{
SEPARACIÓN DE LOS EFECTOS EPISTÁTICOS, ADITIVOS Y DOMINANTES EN MAÍZ, EN MÉXICO1
}

\author{
Mariano Mendoza ${ }^{2}$, Alfonso López ${ }^{3}$, Sergio A. Rodríguez ${ }^{3}$, Carlos De León ${ }^{4}$, Dan Jeffers ${ }^{4}$
}

\section{RESUMEN}

Separación de los efectos epistáticos, aditivos y dominantes en maíz. Se cruzó una línea $\mathrm{P}_{1}$ moderadamente resistente con otra línea susceptible al achaparramiento del maíz y se obtuvo la F1, F2 y retrocruzas a ambos progenitores $\left[R C\left({ }_{\mathrm{P} 1}\right)\right.$ y $\left.R C\left({ }_{\mathrm{P} 2}\right)\right]$; estas progenies fueron evaluadas en dos fechas de siembra en Veracruz en 1999, bajo un diseño de medias generacionales. Los resultados señalan que los efectos epistáticos fueron significativos para el achaparramiento del maíz, no obstante, los efectos génicos dominantes fueron más grandes e importantes que los efectos aditivos y epistáticos. Los datos observados indican que son pocos genes mayores los que gobiernan la resistencia al achaparramiento. El híbrido (F1) resultó con alta tolerancia indicando que existe dominancia, asimismo, se observó heterosis para la resistencia al achaparramiento. Estos resultados sugieren algún programa de selección recurrente para mejorar esta característica o bien un programa de retrocruzas que permita incorporar resistencia a germoplasma susceptible.

\begin{abstract}
Separation of epistatic, additive and dominant effects on corn. The inheritance of host to stunt maize was studied under natural field conditions. Inbred lines $\mathrm{P}_{1}$ (moderately resistant) and $\mathrm{P}_{2}$ (susceptible) and their $\mathrm{F}_{1}, \mathrm{~F}_{2},\left[\mathrm{BC}\left(\mathrm{P}_{1}\right)\right.$ and $\mathrm{BC}\left(\mathrm{P}_{2}\right)$ ] progenies were evaluated at two seasons at sea level, in Veracruz in 1999. Significant epistatic effects were noted to the resistance to stunt corn; however, the dominant genetics effects were greater and more important than additive and epistatic effects. The data showed that resistance to stunt corn was controlled by only a few major genes. The $\mathrm{F}_{1}$ hybrid showed high tolerance, indicating dominance and mid-parent heterosis for resistance was also observed. The data indicate that a back-crossing procedure can be used to transfer the resistance factors into elite lines. Some form of recurrent selection in populations having a degree of resistance also is suggested.
\end{abstract}

\section{INTRODUCCIÓN}

En México se estiman grandes pérdidas por la pudrición de tallo y grano causado por Fusarium. Este hongo aparece primero como una coloración salmón pálido en el pedicelo o casquete de la punta de los granos. Eventualmente, los granos infectados muestran un crecimiento de moho polvoso de color rosáceo, compuesto por grandes números de esporas o conidias. Las líneas puras difieren en su resistencia a esta enfermedad. Las líneas puras susceptibles tienen "estigmas cortados" o granos reventados. Tales líneas transmiten tegumentos débiles de la semilla a sus híbridos y tienden a ser susceptibles a la pudrición del grano por Fusarium. En el tallo el hongo penetra cerca de la ocrana de la planta o a través de los nudos. Las plantas afectadas se vuelven cafés y los tejidos se reblandecen en la planta inferior del tallo. Conforme la enfermedad avanza, el tejido medular se distribuye y sólo quedan en el tallo las fibras de los vasos que conducen el agua. Las líneas puras difieren considerablemente en su resistencia a Fusarum, por lo

\footnotetext{
1 Recibido para publicación el 20 de junio del 2001.

2 Instituto Tecnológico Agropecuario No. 33. Roque, Celaya, Gto., México. Tel/fax (461)16362. Email: mmendoza66@hotmail.com.

3 Depto. de Fitomejoramiento, Universidad Autónoma Agraria Antonio Narro, Buenavista, Saltillo, Coah., México. Tel/fax (84)177361;

4 Centro Internacional de Mejoramiento de Maíz y Trigo (CIMMYT). Apdo. Postal 6-641, Col. Juárez, Deleg. Cuauhtémoc 06600 México, D. F. México.
} 
que el mejor método de control es el uso de híbridos. Por otro lado, las enfermedades ocasionadas por Mollicutes presentan síntomas muy variables y los más comunes son la pérdida de color debido a la reducida producción de clorofila, el enanismo, entrenudos cortos, y bajos rendimientos, entre otros. La incidencia del achaparramiento en maíz se está incrementando causando pérdidas severas a los productores de maíz del trópico y subtrópico (Bradfute et al. 1981). Actualmente, la enfermedad se presenta en forma endémica, llegando a causar del 70 al $100 \%$ de pérdida de siembras afectadas (Waquil et al. 1997, De León et al. 1999). La enfermedad se ha reportado en incidencias de leves a severas en el sur de Estados Unidos, México, países de Centro América y Caribe, Colombia, Venezuela, Ecuador, Brasil, Perú, Paraguay y Norte de Argentina. En condiciones de campo, normalmente el achaparramiento se presenta en combinación con el virus del rayado fino (MRFV), el cual puede causar pérdidas en un promedio de $40-50 \%$ en variedades adaptadas, mientras que los genotipos introducidos o desarrollados pueden sufrir hasta el $100 \%$ de pérdidas (Gámez 1983). El achaparramiento se describió inicialmente en California y Texas (Alstatt 1945). Posteriormente, Maramorosch (1955), usando protección cruzada entre plantas con achaparramiento colectadas en Texas y en el altiplano de México, encontró dos razas del llamado virus del achaparramiento a las que determinó como la raza Río Grande y la Mesa Central. En 1968 (Maramorosch et al.), demostraron la asociación de estructuras parecidas a micoplasmas en plantas con la enfermedad. Fue en 1973 que Davis encontró la presencia de espiroplasmas en plantas de maíz con achaparramiento tipo Río Grande. En 1981, en trabajos iniciados en 1979 por Bascopé y Galindo, se aclaró esta situación al determinar que los dos tipos de achaparramiento eran causados por diferentes patógenos, uno causado por espiroplasmas (CSS) y otro por organismos parecidos a micoplasmas. Estos resultados fueron confirmados por Nault (1980). Ambos agentes son procariotes de la Clase Mollicutes. Dentro del complejo del achaparramiento, se incluyó el virus del rayado fino. A la fecha aún existe confusión en los síntomas del complejo de achaparramiento, ya que la infección puede ser causada por la acción combinada de espiroplasmas (CSS), fitoplasma (MBS), y virus del rayado fino (MRFV) u otro patógeno.

Los hospederos naturales de estos patógenos son unos cuantos géneros, incluyendo Zea, Tripsacum y Euchlaena spp (teosintle). Markham et al. (1977) mencionan que sorgo y zacate Johnson pueden albergar el CSS. Las diferencias en rango de hospederos son que Zea perennis es inmune a MBS y MRFV, pero susceptible a CSS. Zea diploperennis es susceptible a CSS y MRFV, pero inmune a MBS. MRFV puede infectar al
Tripsacum australe y a Roettboellia exaltata. MBS tiene como hospederos a maíz y teosintle, Tripsacum no ha mostrado susceptibilidad. En cuanto, al virus rayado fino (MRFV) está restringido a maíz, teosintle y Tripsacum.

Los patógenos que han sido asociados con el achaparramiento son los espiroplasmas (CSS), fitoplasmas del enanismo arbustivo del maíz (MBS), y el virus rayado fino (MRFV).

El espiroplasma es de morfología filamentosa helicoidal que mide $0,2-0,25 \times 3-15$ micras, tiene movimiento contráctiles, no posee pared celular, son frecuentes en el floema de las plantas. En la planta el patógeno se encuentra limitado al floema, translocándose a sitios activos de crecimiento. En maíz, existe clorosis en bandeado fino extendiéndose desde la base hacia la punta de la hoja. La coloración roja, sí se presenta, es menor que en el caso del virus rayado. En zonas frescas la clorosis es menos intensa, contrasta menos con el verde de la hoja, más bien es un amarillamiento, no en bandas sino expandido en toda la lamina de la hoja. Los síntomas se presentan a los 1643 días después de la inoculación (Nault 1980).

Los fitoplasmas son unicelulares, sin pared celular (pleomórficos), sin membrana nuclear (procariontes) y sensibles a tetraciclinas. Al igual que los espiroplasmas, el control químico de estos organismo no resulta viable debido a que la tetraciclina solamente es bactericida y no elimina completamente estos patógenos. Tienen similitud con algunos virus en síntomas inducidos, localización limitada al floema y trasmisión circulativa-propagativa por chicharritas. En forma general, induce amarillamientos uniformes "escobas de brujas", enanismos, deformación de flores y frutos y coloraciones diversas (Lozoya 1989). El enrojecimiento de la hoja o color púrpura, comienza en los extremos de las hojas extendiéndose a toda la lámina. En plantas viejas y secas, las hojas son quebradizas. Los síntomas aparecen entre los nueve y 19 días después de la infección, dependiendo de la temperatura en igual manera que CSS (Nault 1980).

En el virus del rayo fino los viriones son isométricos de 22 a $30 \mathrm{~nm}$ de diámetro, las partículas sedimentan en dos componentes, un componente contiene cápsidos vacíos de proteína y el otro contiene la nucleoproteína infectiva. Primeramente puede aparecer un aclaramiento (clorosis) en los márgenes de las hojas jóvenes o una coloración roja que luego se torna al púrpura y rojo quemado apagado (Nault 1980). La planta queda enana "chaparra", por reducción de la longitud de los entrenudos. Pero en infecciones más leves o más 
tardías pueden crecer a altura casi normal. Algunas veces existe proliferación de brotes y/o de chilotes estériles que se forman en casi todas las axilas. Los síntomas se desarrollan entre los ocho y 14 días después de la inoculación; si las plantas son infestadas en edad avanzada los síntomas tienden a desaparecer (Gámez 1983).

Entre los hospederos del vector, el $D$. maidis tiene preferencia por Zea mays, Z. diploperennis y Z. perennis. Otros hospederos menos preferidos son Tripsacum dactyloides, T. lanceolatum y E. mexicana. Los hospederos que permiten completar su ciclo son Z. mays con sus subespecies mays var. parviglumis, arviglumis var. huehuetenangensis y mexicana, Z. diploperennis y $T$. dactyloides.

De acuerdo con lo antes señalado, se plantea como objetivo del presente trabajo determinar los efectos génicos y el número de genes involucrados en la resistencia al achaparramiento del maíz.

\section{MATERIALES Y MÉTODOS}

\section{Material genético}

De la población Molcates desarrollada por el Instituto Mexicano del Maíz ubicado en la Universidad Autónoma Agraria Antonio Narro (UAAAN), se seleccionaron líneas resistentes y/o tolerantes y susceptibles al achaparramiento. Plántulas de esta población habían sido evaluadas el ciclo anterior mediante inoculaciones artificiales depositando seis a ocho chicharritas infectivas por plántula. En el ciclo primavera-verano 1998A se hicieron las cruzas RxR, RxS y SxS. Para el ciclo otoño-invierno 1998B, las F1 obtenidas se avanzaron a F2 por autofecundación y los progenitores se retrocruzaron al progenitor uno $[\mathrm{RC}(\mathrm{P} 1)]$ y al progenitor dos [RC(P2)] polinizando las F1 correspondientes con el progenitor uno y progenitor dos, respectivamente. En el ciclo 1999A todos los progenitores y cruzas se incrementaron obteniéndose las generaciones $\mathrm{P} 1, \mathrm{P} 2, \mathrm{~F} 1, \mathrm{~F} 2$, $\mathrm{RC}(\mathrm{P} 1)$ y $\mathrm{RC}(\mathrm{P} 2)$, necesarias para este estudio.

\section{Descripción del área de estudio}

La evaluación de las medias generacionales se llevó a cabo en dos fechas de siembra en la localidad de Villa Ursulo Galván, Ver. en el ciclo 1999B. Esta localidad tiene suelo tipo Feozen y Vertisol, a 19²4'17' lat. N, $102^{\circ} 46^{\prime} 28^{\prime \prime}$ long. E, $8 \mathrm{msnm}$, y temperatura y precipitación media anuales de $25,8^{\circ} \mathrm{C}$ y $1017,7 \mathrm{~mm}$, respectivamente, con lluvias abundantes en verano y principios de otoño, con un clima tropical húmedo.

\section{Trabajo de campo}

La fertilización se realizó manualmente aplicando $200 \mathrm{~kg}$ de fertilizante 15-15-15, suplementado con 120 $\mathrm{kg}$ de superfosfato triple de calcio y $240 \mathrm{~kg}$ de urea. Las aplicaciones de riego e insecticidas se hicieron cuando se consideró necesario. El experimento se estableció con dos repeticiones, en parcelas de un surco con 4,20 $\mathrm{m}$ de longitud y una distancia entre plantas de $0,20 \mathrm{~m}$, para obtener una densidad de aproximadamente 60000 plantas/ha. La incidencia de achaparramiento en los diferentes genotipos se realizó contando plantas individuales en los diferentes grados de severidad y el número total de plantas por parcela cuando el cultivo estaba en la etapa de llenado de grano. En esta etapa se observaron claramente los diferentes grados de achaparramiento. Para ello se utilizó la escala descrita por Grogan y Rosenkranz (1968): Grado 1: Sin síntomas; Grado 2: Síntomas visibles en las hojas en $1 / 4$ de la planta. Achaparramiento no evidente; Grado 3: Síntomas en las hojas en la mitad de la planta. Achaparramiento moderado; Grado 4: Síntomas en las hojas en $3 / 4$ de la planta. Achaparramiento severo; Grado 5: Más de $3 / 4$ de la planta con síntomas en las hojas y achaparramiento severo. Se consideraron plantas tolerantes o resistentes las situadas en los grados uno y dos y como plantas susceptibles aquellas en las escalas tres, cuatro y cinco. Normalmente, las plantas en grados cuatro y cinco presentan mal desarrollo de espiga, esterilidad y poco o nada de formación de semilla. La variable cobertura de mazorca se tomó antes de la cosecha realizándose un conteo de las plantas con problemas, posteriormente se estimó el porcentaje de la misma en base al número total de plantas cosechadas, en relación a $F u$ sarium fue tomada una vez realizada la cosecha contando el número de mazorcas con síntomas, de la misma manera se expresó en por ciento, la variable prolificidad se refiere a cuantas plantas producen más de una mazorca, también expresada en por ciento; asimismo se tomó el rendimiento de mazorca ajustando la humedad al 15,5 por ciento de humedad.

\section{Diseño experimental}

Se utilizó un diseño de bloques completos al azar, con dos repeticiones en dos fechas de siembra.. Para el análisis de medias generacionales se aplicó el método propuesto por Cavalli (1952) que estima los parámetros y se prueba la aditividad del modelo; la deducción de los coeficientes de los parámetros se basó en lo descrito por Márquez (1985). Para estimar estos coeficientes 
se asume que se cruzan los progenitores $\mathrm{P}_{1}(\mathrm{AA})$ y $\mathrm{P}_{2}$ (aa), donde el total de su progenie $F_{1}$ será Aa. Esta se avanza a $\mathrm{F}_{2}$ por autofecundación resultando una frecuencia genotípica de $1 / 4$ Aa, $1 /{ }_{2}$ aa y $1 / 4$ AA. Para la retrocruza, la $\mathrm{F}_{1}$ se cruza hacia el padre con el alelo favorable $\left(\mathrm{P}_{1}\right)$ y la progenie será $1 / 2$ Aa y $1 / 2$ Aa $\left[\mathrm{BC}\left({ }_{\mathrm{P} 1}\right)\right]$, pero si la retrocruza es hacia el $\mathrm{P}_{2}$ la estructura es $1 / 2$ aa $\mathrm{y}^{1 / 2}$ Aa $[\mathrm{BC}(\mathrm{P} 2)]$.

\section{Estimación de la heterosis y depresión endogámica}

La heterosis promedio de los progenitores se calculó usando la fórmula ${ }^{1}$ y la depresión endogámica con la fórmula ${ }^{2}$ descrita por Vázquez (1999):

$$
\begin{aligned}
& \text { 1Heterosis }=\frac{\left[F_{1}-1 / 2\left(P_{1}+P_{2}\right)\right]}{1 / 2\left(P_{1}+P_{2}\right)} \\
& \text { 2Depresión endogámica }=\frac{\left.\left[F_{1}-F_{2}\right)\right]}{\left.F_{1}\right)} * 100
\end{aligned}
$$

\section{Número de genes}

Para estimar el número de genes involucrados se usaron las medias, las $\sigma^{2}$ de F1, F2 y de la retrocruza, para ser sustituídas en la fórmula descrita por Castle (1921) y utilizada por Dollinger et al. (1970) y Vázquez (1999):

$$
\text { No. de factores }=\frac{D^{2}}{8\left(\sigma^{2} F_{2}-\sigma^{2} F_{1}\right)}
$$

Donde: $\mathrm{D}=$ diferencia entre las medias de los progenitores, $8=$ constante, $\sigma^{2} F_{1}=$ varianza de la $F_{1} y$ $\sigma^{2} \mathrm{~F}_{2}=$ varianza de la $\mathrm{F}_{2}$.

Cuadro 1. Promedio de las variables en estudio a través de un diseño de medias generacionales.

\begin{tabular}{lllrrr}
\hline Gen & $\begin{array}{l}\text { PCA } \\
(\%)\end{array}$ & $\begin{array}{l}\text { MC } \\
(\%)\end{array}$ & \multicolumn{1}{c}{$\begin{array}{c}\text { MCF } \\
(\%)\end{array}$} & $\begin{array}{c}\text { Maz/100 } \\
\text { plantas }\end{array}$ & $\begin{array}{c}\text { Rendimiento } \\
(\mathbf{t} / \mathbf{h a})\end{array}$ \\
\hline P1 & 37,50 & 0,00 & 22,78 & 124,48 & 5,1469 \\
P2 & 52,18 & 0,00 & 25,86 & 101,72 & 2,9356 \\
F1 & 24,74 & 1,28 & 5,34 & 107,48 & 5,3756 \\
F2 & 30,00 & 3,57 & 12,28 & 124,02 & 3,1263 \\
RC(P1) & 26,56 & 0,00 & 18,57 & 92,77 & 5,9094 \\
RC(P2) & 43,78 & 2,94 & 13,66 & 132,77 & 3,9269 \\
\hline
\end{tabular}

MCF: mazorcas con Fusarium, MC: mala cobertura de mazorca, PCA: plantas con achaparramiento.

\section{RESULTADOS Y DISCUSIÓN}

El porcentaje de plantas con síntomas de achaparramiento fue de $37,50 \%$ para el progenitor tolerante y $52,18 \%$ para el susceptible (Cuadro 1). Debido a la presencia de dominancia del carácter, en la F1 se obtuvo una reducción en los valores de incidencia hasta un $24,74 \%$, respuesta similar se encontró en la retrocruza con el progenitor tolerante $(26,56 \%)$. Lo contrario se observa que cuando el cruzamiento se hace hacia el padre susceptible $(43,75 \%)$. La incidencia de mazorcas con Fusarium es ligeramente menor en el progenitor tolerante; no obstante, en la F1 también se refleja una sobredominancia al tener un valor de 5,34 \%, en la F2 se observaron $12,28 \%$ y $18,57 \%$ para la retrocruza al padre tolerante y $13,66 \%$ cuando se utilizó el padre susceptible. En cobertura de mazorca no se presentaron problemas para los progenitores, al igual que en la retrocruza con el padre tolerante. Los valores observados no fueron de importancia económica, ya que los datos varían entre 1,28 y $3,57 \%$. Respecto a prolificidad de mazorca se encontró una diferencia de $23 \%$ hacia el progenitor tolerante, resultando una dominancia parcial en la F1. En la F2 y en la retrocruza hacia el progenitor susceptible se observó un incremento importante para esta variable. Sin embargo, el tamaño de mazorca no fue significativo al estimar el rendimiento. Los resultados para ésta última característica indican una diferencia entre ambos progenitores de $2213 \mathrm{t} / \mathrm{ha}$ indicando que el material susceptible fue fuertemente afectado por la incidencia de achaparramiento, tanto por la cantidad como por el tamaño de las mazorcas por planta (Cuadro 1).

Los efectos genéticos promedios se presentan en el Cuadro 2. Estos resultados indican que en las variables plantas con achaparramiento, mazorcas con Fusarium,

Cuadro 2. Efectos promedios de los parámetros genéticos. Pro-

\begin{tabular}{|c|c|c|c|c|c|}
\hline Efecto & PCA & $\begin{array}{l}\text { Mazorcas } \\
\text { c/fusarium }\end{array}$ & $\begin{array}{l}\text { Cobertura } \\
\text { mazorca }\end{array}$ & $\begin{array}{l}\text { Maz. } x \\
100 \text { plantas }\end{array}$ & $\begin{array}{l}\text { Rendimiento } \\
(\mathbf{t} / \mathbf{h a})\end{array}$ \\
\hline $\mathrm{a}$ & $-0,55$ & $-0,15$ & 0,00 & $-3,62$ & 1,12 \\
\hline d & $1,74^{*}$ & 2,71 & $-3,69$ & $39,30 *$ & $16,46^{*}$ \\
\hline $\mathrm{a} \times \mathrm{a}$ & 1,60 & 1,92 & $-2,58$ & $-5,00$ & 7,17 \\
\hline $\mathrm{a} \times \mathrm{d}$ & $-1,82$ & 1,50 & $-1,98$ & $-72,76^{*}$ & 1,72 \\
\hline $\mathrm{d} \times \mathrm{d}$ & $-1,84^{*}$ & $-3,30$ & 1,62 & $-34,92$ & $-7,98$ \\
\hline
\end{tabular}
genitor resistente $\mathrm{x}$ progenitor susceptible al achaparramiento.

PCA, plantas con achaparramiento, MCF: mazorcas con Fusarium, MC: mala cobertura de mazorca; a: efecto aditivo; d: efecto dominante; a $\mathrm{x}$ a: efecto aditivo por aditivo; a $\mathrm{x}$ d: efecto aditivo por dominante; $\mathrm{d} \mathrm{x}$ d: efecto dominante por dominante. 
prolificidad de mazorcas y rendimiento, los efectos de tipo dominante fueron más importantes que los efectos aditivos. En relación a los efectos epistáticos, el aditivo $\mathrm{x}$ aditivo $\mathrm{y}$ aditivo $\mathrm{x}$ dominante resultaron ser más importantes en las características de mazorcas con Fusarium y rendimiento. En relación a los efectos epistáticos, el aditivo $\mathrm{x}$ aditivo $\mathrm{y}$ aditivo $\mathrm{x}$ dominante resultaron ser más importantes en las características de mazorcas con Fusarium y rendimiento. Para el carácter de cobertura de mazorca se detectó presencia de efecto dominante $\mathrm{x}$ dominante $\mathrm{y}$ para plantas con achaparramiento el efecto aditivo $\mathrm{x}$ aditivo. Para rendimiento, todos los efectos fueron positivos a excepción de la interacción dominante $\mathrm{x}$ dominante.

En el Cuadro 3, se muestra que el achaparramiento, mazorcas con Fusarium, cobertura de mazorca y prolificidad no presentaron depresión, por lo que no se afecta el número de mazorcas en la F2. Para el carácter de plantas con achaparramiento los valores se presentaron en forma similar. En mazorcas con Fusarium, la media muestra una depresión de -129,96 mientras que la cobertura de mazorca presenta una media de $-178,91$. Para el carácter rendimiento, éste se reduce en 41,84\% entre las generaciones F1 y F2 al utilizar la semilla F1 al siguiente ciclo (F2). Golovochonko y Glukhovtsev (1997); indican una disminución en rendimiento de $22,7 \%$. Se debe considerar que además de la depresión endogámica en la F1, en el presente estudio se utilizó un progenitor resistente y uno susceptible al achaparamiento, por lo que la depresión es más severa. Beck et al. (1990) indican una heterosis positiva y significativa para este carácter. Fasoulas (1986) menciona que si no existe reducción en el vigor en una F2, teóricamente el vigor híbrido o heterosis está determinado por la acción equilibrada de genes codominantes y semidominantes. En el presente trabajo, la heterosis fue expresada por la heterocigosidad. La variable de plantas con síntomas de achaparramiento presenta dominancia negativa de 44,83 (heterosis negativa), indicando que existió una reducción de los síntomas. Esto se puede atribuir a que existe resistencia y/o tolerancia al achaparramiento en

Cuadro 3. Heterosis y depresión endogámica entre dos progenitores de maíz.

\begin{tabular}{lrrrrr}
\hline Parámetro & $\begin{array}{c}\text { PCA } \\
(\boldsymbol{\%})\end{array}$ & $\begin{array}{c}\text { MCF } \\
(\boldsymbol{\%})\end{array}$ & $\begin{array}{r}\text { MC } \\
(\boldsymbol{\%})\end{array}$ & $\begin{array}{c}\text { Maz/ 100 } \\
\text { plantas }\end{array}$ & $\begin{array}{c}\text { Rendimiento } \\
(\mathbf{t} / \mathbf{h a})\end{array}$ \\
\hline Heterosis & $-44,83$ & $-78,04$ & 0,00 & 29,34 & 33,02 \\
Depresión E & $-21,26$ & $-129,96$ & $-178,91$ & $-43,30$ & 41,84 \\
Genes & 0,66 & 0,12 & 0,10 & 0,20 & 5,40 \\
\hline
\end{tabular}

PCA, plantas con achaparramiento, MCF, mazorcas con Fusarium, $\mathrm{MC}$, mala cobertura mazorca. algunos de los progenitores, o en ambos (Narro-León y Miranda 1997). Estos mismos autores indican una heterosis de $-21,71 \%$, menor al que se obtuvo en este trabajo. Johnson (1971), en un estudio a la resistencia genética al mosaico del enanismo del maíz, determinó una heterosis del progenitor medio para la resistencia. En mazorcas con problemas de Fusarium, la heterosis fue negativa, lo que sugiere dominancia negativa, o una reducción en el daño. En la cobertura de mazorca la heterosis fue nula. En la característica prolificidad o número de mazorcas por 100 plantas se encontró heterosis de $29,34 \%$, que influyó en el rendimiento, ya que ésta última característica presentó dominancia (heterosis) obteniéndose una mayor producción; al respecto, Golovochenko y Glukhoutsev (1997) encontraron un 33,3\% de heterosis para rendimiento. Sin embargo, la presencia de una alta dominancia y epistasis no siempre indica alta heterosis (Khotyleva y Tarutina 1997).

Las estimaciones promedios genéticas indican que para mazorcas con Fusarium, cobertura de mazorca y prolificidad están gobernados por un par de genes. Para rendimiento se estimaron cinco pares de genes, lo que sugiere que un programa de hibridación o de retrocruzas sea el más adecuado para mejorar estas características. Boling y Grogan (1965) al determinar la herencia de la resistencia a la pudrición de la mazorca por Fusarium, obtuvieron estimaciones del número de pares génico a la resistencia de aproximadamente 1,47. Con respecto a la enfermedad del achaparramiento se encontró que un par de genes controlan la resistencia, no obstante, Loesch y Zuber (1973), estudiando la resistencia al virus del mosaico del enanismo del maíz, encontraron que la resistencia a esta enfermedad está condicionada por más de un gen y que la manifestación de la enfermedad dependía de condiciones ambientales.

\section{LITERATURA CITADA}

ALSTATT, G. E. 1945. A new corn disease in the Rio Grande Valley. Plant Dis. Reptr. 29:533-534.

BASCOPÉ, B., O.; GALINDO, J. 1981. Naturaleza micoplásmica de la raza "Mesa Central del achaparramiento” del maíz. Fitopatología (México) 16:28-33.

BECK, D. L.; VASAL, S. K. ; CROSSA, J. 1990. Heterosis and combining ability of CIMMYT's tropical early and intermediate maturity maize (Zea mays L.) germoplasm. Maydica 35:279-285.

BOLING, M. B; GROGAN, C. O. 1965. Gene action affecting host resistance to Fusarium ear rot of maize. Crop Sci. 5(4):305-307. 
BRADFUTE, O. E.; TSAI, J. H.; GORDON, D. T. 1981. Corn stunt spiroplasma and viruses associated with a maize disease epidemic in Southerm Florida. Plant Disease 65:837-841.

CASTLE, W. E. 1921. An improved method of estimating the number of genetic factors concerned in cases of blending inheritance. Science. 54:223.

CAVALLI, L. 1952. An analysis of linkage in quantitative inheritance. In: Rieve, E.; Waddington, C. eds. Quantitative inheritance. London, HMSO. p. 135-144.

DE LEÓN, C.; NARRO, L.; RUÍZ, C; JEFFERS, D; SALAZAR, F ; ARIAS, M.P. 1999. Avances en la selección de resistencia genética al achaparramiento del maíz. Ascolfi Informa (Colombia) 25:38-40.

DOLLINGER, E. J.; FINDLEY, W. R.; WILLIAMS, L. E. 1970. Resistance of inheritance to maize dwarf mosaic virus in maize. Crop Sci. 10: 412-415.

FASOULAS, A. 1986. The aplication of genetics to plant breeding. Pub. No. 8. Dept. Gen. Plant Breeding. Aristotelian Univ. of Thessaloniki, Greece. p. 23-25.

GÁMEZ, R. 1983. The ecology of maize rayado fino virus in the American tropics. Plant virus epidemiology. p. $267-$ 275 .

GOLOVOCHENKO A. P.; GLUKHOVTSEV, V. V. 1997. Heterosis and inheritance of productivity in reduplicated generations of spring wheat hybrids. In: Book of Abstracts. The genetics and exploitation of heterosis in crops. An internatl. symp. Mexico, D. F. Mexico. p. 318.

GROGAN, C. O.; ROSENKRANZ, E. E. 1968. Genetics of host reaction to corn stunt virus. Crop Sci. 8:251-254.

JOHNSON, G. R. 1971. Analysis of genetic resistance to maize dwarf mosaic disease. Crop Sci. 11:23-24.

KHOTYLEVA, L. V.; TARUTINA, L. A. 1997. Nonallelic interations and heterosis in corn. In: Book of Abstracts.
The genetics and exploitation of heterosis in crops. An internatl. symp. Mexico, D. F. México. p. 146.

LOESCH, P. J.; ZUBER, M. S. 1973. Inheritance of resistance to maize dwarf mosaic virus. Crop Sci. 12:350-352.

LOZOYA, S. H. 1989. Diagnóstico de enfermedades de plantas y bibliografía sobre fitopatología. Universidad Autónoma de Chapingo, México. pp. 13-23.

MARAMOROSCH, K. 1955. The occurrence of two distinct types of corn stunt in Mexico. Plant Dis. Reptr. 39:896898.

MARAMOROSCH, K.; SHIKATA, E.; GRANADOS, R. R. 1968. Structures resembling mycoplasma in diseased plants and insect vectors. Trns. New York Acad. Sci. II, 30:841-845.

MARKHAM, P. G.; TAUSEND, R.; PLASKITT, K.; SAGLIN, P. 1977. Transmission of corn stunt to dicotyledoneous plants. Plant Dis. Reptr. 61:342-345.

MÁRQUEZ, S. F. 1985. Genotecnia vegetal: métodos, teoría resultados. México, Ed. AGT. p. 92.

NARRO - LEÓN, T. P.; MIRANDA, J. B. 1997. Heterosis for resistance to virus, phytoplasmas and spiroplasma in maize. In: Book of abstracts. The genetics and exploitation of heterosis in crops. An internatl. symp. Mexico, D. F. México. p. 178.

NAULT, L. R. 1980. Maize bushy stunt and corn stunt: a comparison of disease symptoms, pathogen host ranges, and vectors. Phytopathology. 70:659-662.

VÁZQUEZ, M. 1999. Efectos genéticos para calidad fisiológica de semilla, características agronómicas y rendimiento en siete líneas de maíz. Tesis Doctoral. Fitomejoramiento, UAAAN. 257 p.

WAQUIL, J. M.; OLIVEIRA, E. ; PINTO, N.F.J. A. 1997. Incidencia de cigarrinha, enfezamento e virose em milho. Ann. IV Seminario Cultura de milho "Safrinha". Feb. 6-7, 1997. Assis, S. P. Brazil. 